\title{
Histopathological changes of reproductive organs of goats immunized with Corynebacterium pseudotuberculosis killed vaccine
}

\begin{abstract}
Caseous lymphadenitis (CLA) caused by Corynebacterium pseudotuberculosis is characterized by the development of abscesses, mainly in superficial and internal lymph nodes, visceral and reproductive organs in small ruminants. This study aims to examine the histopathological changes in reproductive organs of goats immunized with killed vaccine of C. pseudotuberculosis. In this study, twenty four (24) clinically healthy bucks and does were divided into four groups A, B, C and D. Animals in groups A and B were immunized with 0.5 and $1 \%$ formalin killed vaccine, respectively; followed by a booster dose. After the booster dose of immunization, groups A, B and $\mathrm{C}$ were challenged with $\mathrm{C}$. pseudotuberculosis at $106 \mathrm{cfu} / \mathrm{ml}$. Goats in group D were immunize and unchallenged and left as control group. All C. pseudotuberculosis infected animals were euthanized humanely 12 weeks post-challenged. Tissue samples such as testes, epididymis, spermatic cord, penis, pituitary gland, mammary gland, vulva, vagina, cervix, uterus, fallopian tube and ovaries were collected for histopathology study. Microscopic examination of all tissues (testes, seminiferous tubules, spermatic cord, penile tissues and the pituitary gland) in the male reproductive organs of the bucks that were inoculated with $2 \mathrm{ml}$ of $0.5 \%$ and $1.0 \%$ of $\mathrm{C}$. pseudotuberculosis killed vaccine showed normal (animals inoculated with $1.0 \%$ ) to mild (animals inoculated with $0.5 \%$ ) histopathological changes when compared with those from group $\mathrm{C}$ which showed varying degrees of histopathological changes $(p<0.01)$ in their various tissues. For the female does, similar histopathological changes were observed for the various tissues examined (ovaries, fallopian tubes, uterine horns, uterine tissues, cervix, vaginal, vulva, mammary glands and the pituitary glands) in which the vaccinated groups A \&B showed a significantly $(\mathrm{p}<0.001)$ less histopathological changes when compared with those in group $C$ that showed varying degrees of histopathological changes in the reproductive organs investigated. This study showed the efficacy of C. pseudotuberculosis killed vaccine protecting against reproductive tissue damages cause by the active infection with the live bacteria in both bucks and does in the study area.
\end{abstract}

Keyword: Caseous lymphadenitis; Corynebacterium pseudotuberculosis; Reproductive organs; Buck; DoeImmunization; Killed vaccine 